\title{
Aggregation-induced emission: right there shining
}

\author{
Nan Jiang ${ }^{1 \dagger}$, Tanxiao Shen $^{1 \dagger}$, Jing Zhi Sun ${ }^{1 *}$ and Ben Zhong Tang ${ }^{1,2,3^{*}}$
}

The emergence of fluorescent probes has enabled researchers to find and observe invisible matter from the investigated object without light emitting. For example, green fluorescent protein (GFP), an invention that received the Nobel Prize for Chemistry in 2008, works as a visible fluorescent reporter to monitor the localization and movement of protein-GFP chimeras, protein-protein interactions, gene expression, and many other biological events in living cells in real time. GFP and its analogs have innovated research techniques and features in biological and life sciences.

A recent breakthrough in these research areas is the discovery of fluorescent probes with characteristics of aggregation-induced emission (AIE) [1]. With their unique emission mechanism and distinct emission behavior from classical luminogens, AIE-active luminogens (AIEgens) have contributed new insights into the photophysical process in organic luminescent materials. AIEgens have also been applied as fluorescent probes in the monitoring of cellular physiological processes, as emitting agents in the fabrication of organic lightemitting diodes, and as light concentrators in the construction of organic/polymer photovoltaic cells [2-5]. In 2016, AIE-based luminescent dots were deemed to be among the four fundamental nanomaterials in supporting the coming "nanolight revolution," and the "virus-sized particles that fluoresced in every color could revolutionize applications from television displays to cancer treatment" [6]. In 2017, Prof. Tang and colleagues received the firstclass prize in the National Natural Science Awards for their distinguished work on the mechanism study, molecular design and preparation, and material applications of AIEgens. In recent years, AIE has become one of the top five major research areas in chemistry and material sciences, according to data on the Web of Science.

The AIE system turns from a faintly emissive to a brightly emissive state when molecular aggregates originate from individual molecules that have a sufficient degree of motion freedoms. When tetraphenylethene (TPE) is used as a representative $[7,8]$, the aggregate formation process must be accompanied by conformation rearrangements of phenyl groups attaching to the central ethylene moiety. A series of AIEgens showing mechanofluorochromic effect have revealed the dependence of emission features (emission peak $\lambda_{\text {em }}$, efficiency $\Phi$, and lifetime $\tau$ ) on the molecular conformation (e.g., dihedral angle change). However, this finding only involves information on the original and terminal states. Since the AIE phenomenon was first reported, limited direct evidence was available to demonstrate the dynamic details of conformational changes.

In a recent work, Zhang et al. [9] contributed a subtle design to expose the analytical actions of intramolecular rotations under spotlight. In this design, a TPE unit is covalently and directly attached to the 3- or 6-position of a $(R)$-1,1'-binaphthyl-unit to derive four different polymers (Fig. 1a). When the polymer chain coils collapse in poor solvent mixtures, the restricted intramolecular rotations in the TPE units induce the fluorescence (FL) emission (P-1, Fig. 1b), which is a common observation for TPE-related molecules and macromolecules. However, the conjugation of TPE with a $(R)$-1,1'-binaphthylunit makes uncommon sense from all of the previous studies. As shown in Fig. 1c, d, the aggregation process or the conformational variation scenario is reflected by the subtle changes of the circular dichroism (CD) spectra of

\footnotetext{
${ }^{1}$ MOE Key Laboratory of Macromolecular Synthesis and Functionalization, Department of Polymer Science and Engineering, Zhejiang University, Hangzhou 310027, China

${ }^{2}$ Department of Chemistry, the Hong Kong Branch of Chinese National Engineering Research Center for Tissue Restoration and Reconstruction and Institute for Advanced Study, The Hong Kong University of Science and Technology, Hong Kong 999077, China

${ }^{3}$ Center for Aggregation-Induced Emission, SCUT-HKUST Joint Research Institute, State Key Laboratory of Luminescent Materials and Devices, South China University of Technology, Guangzhou 510640, China

These authors contributed equally to this paper.

* Corresponding authors (emails: sunjz@zju.edu.cn (Sun JZ); tangbenz@ust.hk (Tang BZ))
} 
a
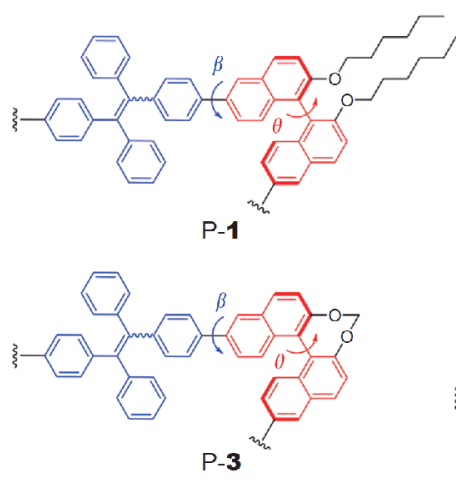

P-3
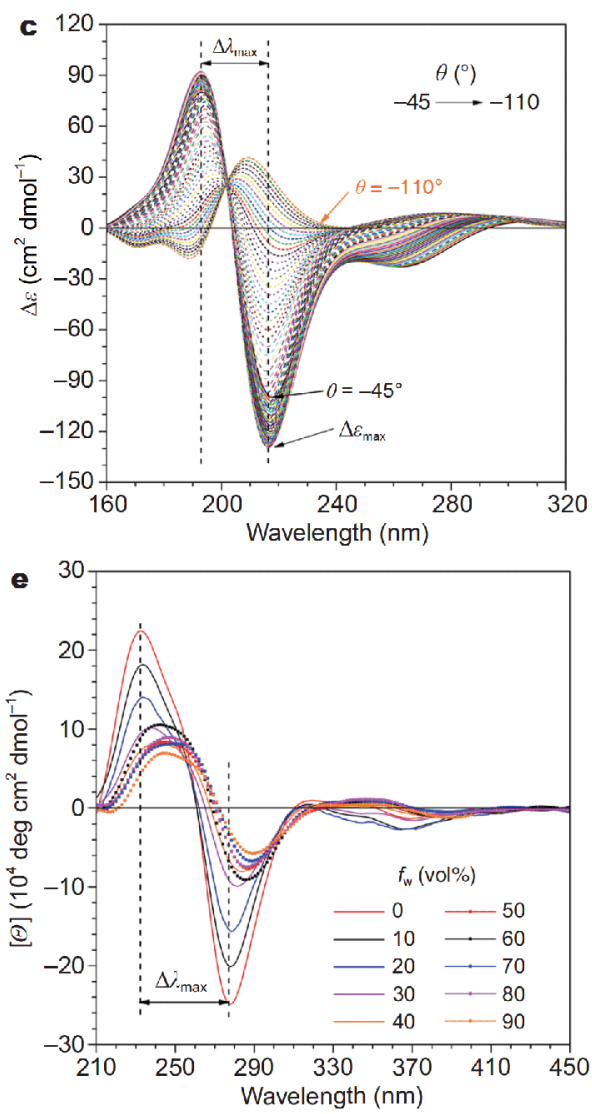

\section{b}

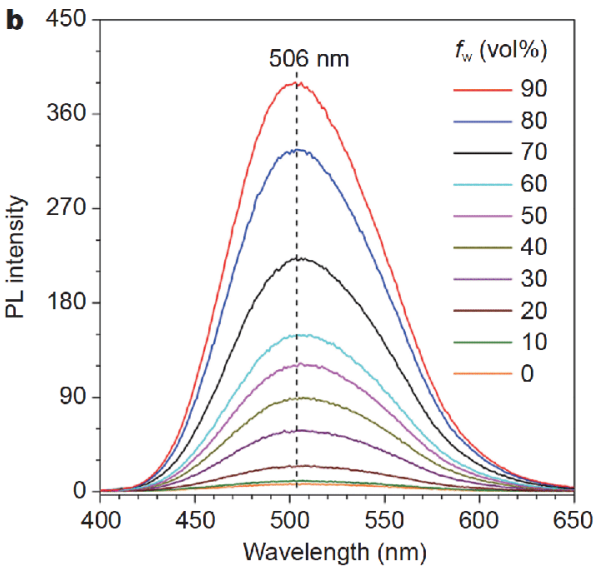

P-4

P-2

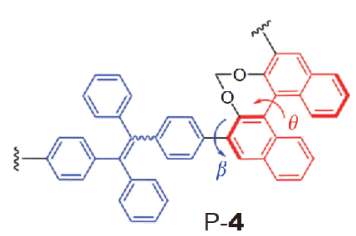

d

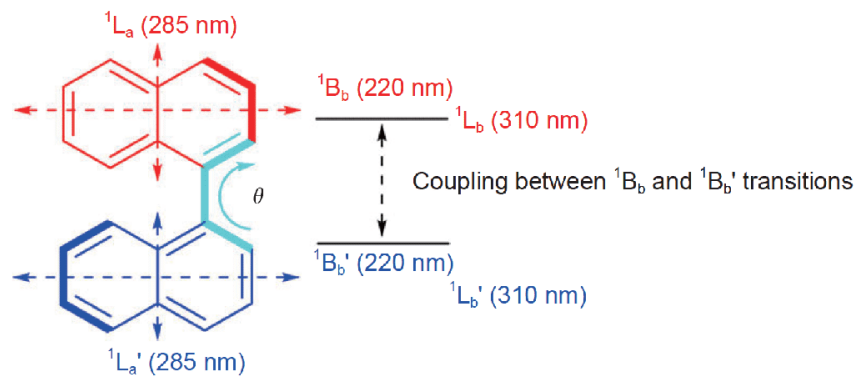

f

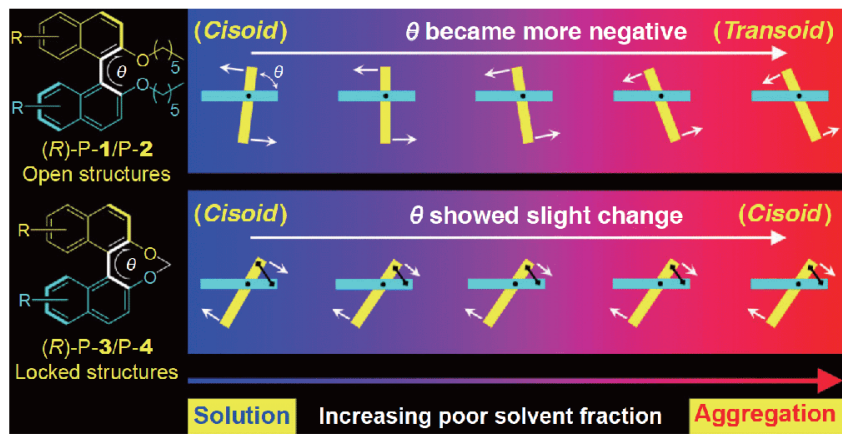

Figure 1 In situ monitoring of molecular aggregation using CD. (a) Chemical structure of designed TPE and 1,1'-binaphthyl-containing polymers P-1 to P-4. (b) Photoluminescence (PL) spectra of P-1 in tetrahydrofuran (THF)/water mixtures with different water fractions $\left(f_{\mathrm{w}}\right)$. Concentration: $10^{-5} \mathrm{~mol} \mathrm{~L}^{-1}, \lambda_{\text {ex }}=355 \mathrm{~nm}$. (c) Simulated CD spectra of $(R)$-1, $1^{\prime}$-binaphthol with $\theta$ varying from $-45^{\circ}$ to $-110^{\circ}$ in $1^{\circ}$ intervals. (d) Schematic of polarization directions of main electronic transition moments and torsion angle $\theta$ of $(R)-1,1^{\prime}$-binaphthyl derivatives. (e) CD spectra. P-1 in THF/water mixtures with different water fractions. Concentration: $10^{-4} \mathrm{~mol} \mathrm{~L}^{-1}$. (f) Schematic representation of conformational change of $(R)-1,1^{\prime}$-binaphthyl moieties during aggregation process. Reproduced with permission from Ref. [9]. Copyright 2018, Nature Publishing Group.

(R)-1,1'-binaphthol unit. As shown in Fig. 1f, a refined model is generated using the dihedral angle $(\theta)$, Davydov splitting width $(\Delta \lambda)$, and negative CD couplet intensity $(\Delta \varepsilon)$ as estimation parameters, combined with theoretical calculation for a number of states, and finally compared with the experimental data (Fig. 1c-e).

According to the experimental and theoretical results, the chiral AIE-active polymers containing 1,1'-binaphthyl 
and TPE units provided an unprecedented model for the real-time and in situ monitoring of the molecular aggregation process. With an "open" structure, polymers P-1 and P-2 exhibited an effect of aggregation-annihilated CD (AACD) signal, and their CD couplet intensity was also largely annihilated by the aggregate formation. On the contrary, the AACD effect was suppressed in both P-3 and P-4 where the "openings" of the binaphthyl moieties were locked by methylene groups. Specifically, the dihedral angle $\theta$ of "open" polymers P-1 and P-2 decreased largely and part of the conformers relaxed from cis oid to trans-oid following the change from the solution to aggregate state. By contrast, the "locked" polymers P-3 and P-4 showed a slightly larger $\theta$ and their cis oid conformations were preserved during the aggregation process. These observations were consistent with the molecular dynamic simulation results and analysis of the change of CD couplet intensity and Davydov splitting during the aggregation process.
In the preceding example, the collapse of the polymer chain was triggered by the addition of a sufficient amount of non-solvent into a real dilute polymer solution. In some cases, the solubility of a macromolecule decreases or even vanishes in the mixture of two good solvents. This phenomenon is called cononsolvency effect, which has been widely applied in the physicochemical, green chemical, and pharmaceutical industries [10-12]. Dynamic light scattering, neutron scattering, isotope labeling, and molecular dynamic simulation techniques have been used in the relevant investigation, but the landscape for cononsolvency effect is elusive. Through the aid of an AIEactive fluorescent probe, a clear picture was drawn by a collaborative study and careful analysis of the emission behaviors of the TPE-crosslinked thermo-sensitive microgels in a nano-confined environment [13].

The molecular design, macromolecular structure, and working mechanism are illustrated in Fig. 2a. The crosslinking networks are formed through the acylhy-
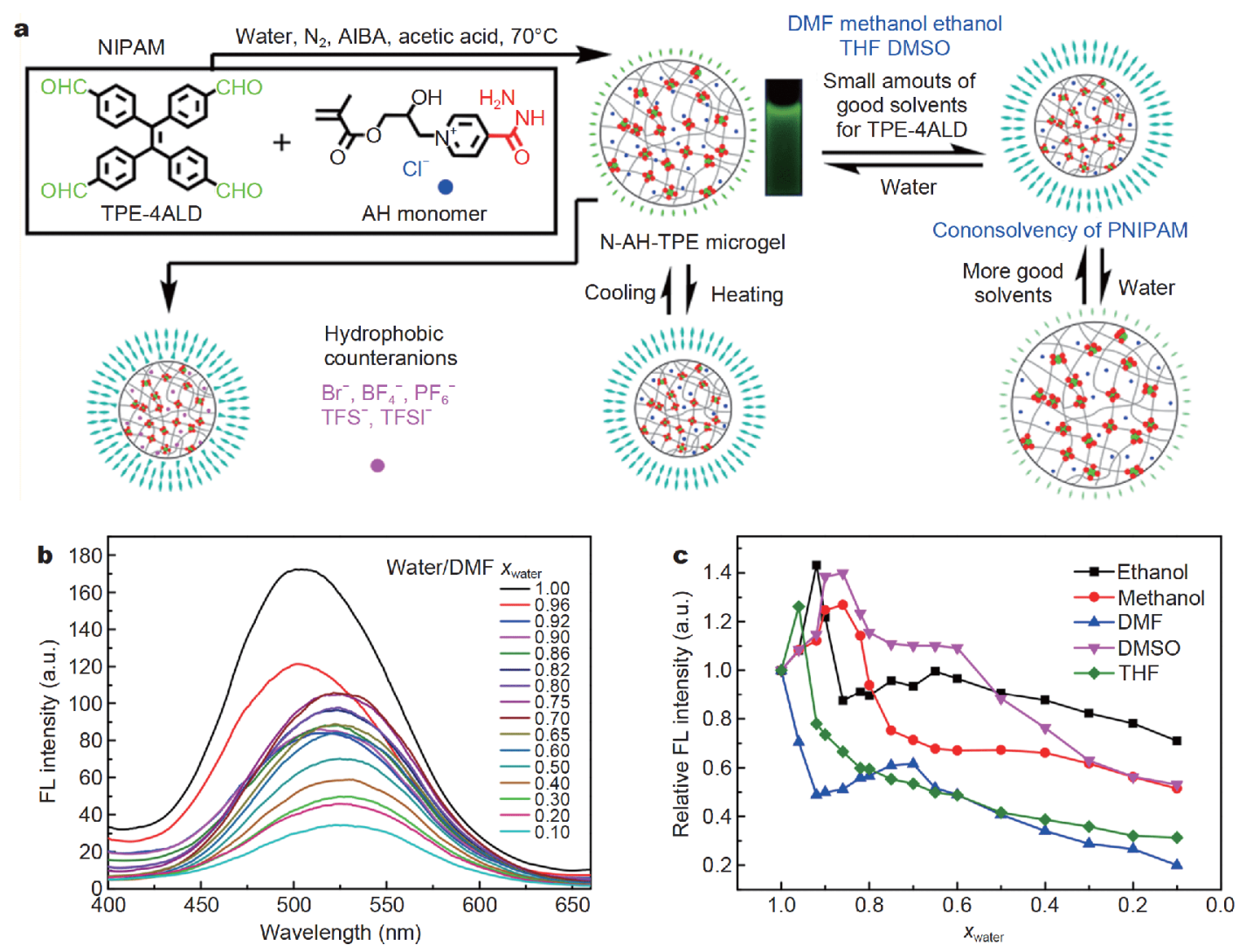

Figure 2 In situ sensing of cononsolvency effect with AIE-active fluorescent probe. (a) Molecular structure of AIE-active probe and monomer, and schematic of cononsolvency behavior of a crosslinked thermo-sensitive microgel. (b) FL spectra of $N$-AH-TPE microgels in water $\left(\mathrm{H}_{2} \mathrm{O}\right) / \mathrm{N}, \mathrm{N}$ dimethylformamide (DMF) mixtures with various water molar fractions $\left(\chi_{\text {water }}\right)$. (c) Plots of emission intensity at maximum emission wavelength of microgels in $\mathrm{H}_{2} \mathrm{O}$ /ethanol (1), $\mathrm{H}_{2} \mathrm{O}$ /methanol (2), $\mathrm{H}_{2} \mathrm{O}$ /DMF (3), $\mathrm{H}_{2} \mathrm{O}$ /DMSO (4), and $\mathrm{H}_{2} \mathrm{O}$ /THF (5) mixtures as a function of water molar fraction. Reproduced with permission from Ref. [13]. Copyright 2018, American Chemistry Society. 
drazone bonds resulting from the condensation of aldehyde groups of TPE-4ALD and acylhydrazine group of 4acylhydrazine-(2-hydroxy-3-(methacryloxypropyl))pyridine hydrochloride ( $\mathrm{AH}$ monomer). By considering the hydrodynamic diameter of $302 \mathrm{~nm}$ for N-AH-TPE microgels in aqueous suspension at $25^{\circ} \mathrm{C}$, we can estimate that one TPE-4AH moiety exists for approximately $394 \mathrm{~nm}^{3}$ for the swollen $\mathrm{N}$-AH-TPE microgels. The selfaggregation of TPE moieties can be ruled out of the analysis because the TPE moieties are confined in the crosslinked network of microgels, and the change in FL intensity could be solely attributed to the variation of the restriction degree of the intramolecular rotations. In aqueous solution, the $\operatorname{poly}(N$-isopropylacrylamide $)$ (polyNIPAM) chains are dissolved and the hydrophobic TPE moieties are almost insoluble. In addition, a TPE moiety is localized at the crosslinking point of the resultant microgel network. In this condition, the intramolecular motions of TPE are restricted. Thus, strong emission could be observed from the microgels.

The TPE moieties are held within the niches of $\mathrm{N}-\mathrm{AH}-$ TPE microgels and cannot diffuse freely even in good solvent. Five organic solvents, namely, DMF, THF, N,Ndimethyl sulfoxide (DMSO), ethanol, and methanol, which are good solvents for TPE and polyNIPAM, as well as miscible with water, have been attempted to examine the cononsolvency effect. Here water/methanol mixture is used as an example. Water and methanol are individually good solvents for polyNIPAM. However, precipitate is observed in a water/methanol mixture with a certain water fraction $(\chi)$, which is a typical phenomenon in cononsolvency. As shown in Fig. 2b, the relative FL of $N$ AH-TPE microgels intensifies with the decrease of $\chi$ from 1.0 to approximately 0.85 (black symbols), which indicates greater restriction of molecular motions (rotations and vibrations) of the TPE units. Correspondingly, the hydrodynamic diameter of the N-AH-TPE microgels in water/methanol mixtures becomes continuously small in the $\chi$ range, suggesting that the co-solvent changes from good to not so good. As $\chi$ decreases from 0.8 to 0.6 , the $\mathrm{FL}$ intensity decreases and the hydrodynamic diameter of the microgels increases monotonously. The solvency improves and the molecular motions are less restricted; these two trends show consistency. Only a narrow $\chi$ window exists between 0.85 and 0.80 , in which the FL intensity diminishes but the hydrodynamic diameter increases. This finding can be explained as follows. As methanol is more hydrophobic than water, the hydrophobicity is weakened when the $\chi$ value is reduced. This condition leads to the activation of the rotations and vi- brations of the phenyl groups on the TPE units, thereby reducing the FL efficiency. However, methanol is a good solvent for polyNIPAM, and the polymer chains can be swollen in the water/methanol mixtures with suitably reduced $\chi$ values. In other words, TPE units and polyNIPAM chains have a distinct sensing ability to hydrophobicity and hydrogen bond formability. Similarly, cononsolvency was observed for polyNIPAM microgels in water-methanol mixtures and other water-miscible polar solvents including THF, DMF, and DMSO (Fig. 2c). These primary achievements imply that AIE-active fluorescent probes can be a general method to investigate the interactions between solvent molecules and polymer chains.

The cononsolvency effect results from mixing two good solvents and leads to movement frozen of polymer chains. The freezing process in a good solvent can also be triggered by temperature change and/or other external stimuli. Sol-gel transition, a typical freezing process of polymer chains, is one of the most important topics in processing and practical application of polymeric materials. Chitosan (CS), a representative polysaccharide, has attracted considerable attention for its application as a resource-renewable and eco-friendly macromolecule [14-16]. In a recently developed technique for the fabrication of CS-based materials and devices, CS alkali ( $\mathrm{LiOH}$ )-urea aqueous solutions have been applied as precursor [17]. After thermal gelation process in a mold and a post-rinsing treatment, the final products can be obtained. Compared with traditional techniques, this method of CS hydrogel preparation has shown advantages such as considerable improvement in hardness, strength, and toughness. In addition, the distinct gelation behavior offers innovative hydrogel design strategies. Thus, the study of gelation possesses high industrial and academic value. However, understanding of the gelation mechanism has been limited for a long time due to the shortage of suitable techniques for monitoring of the in situ gelation process.

In 2016, Wang et al. [18] introduced AIE-active fluorescent probe as an in situ reporter for gelation. As demonstrated in Fig. 3a, TPE units are covalently attached onto the side chain of the polysaccharide, and the trace amount of probe has no effect on the solubility and processability of TPE-modified CS (TPE-CS) due to the high sensitivity of FL technique. After thermal gelation and rinsing treatment, the TPE-CS in the $\mathrm{LiOH}$-urea solution emits strong blue FL under UV light (Fig. 3b). Time-dependent confocal laser scanning FL microscope images reveal details of the gelation process (Fig. 3c). The 
a
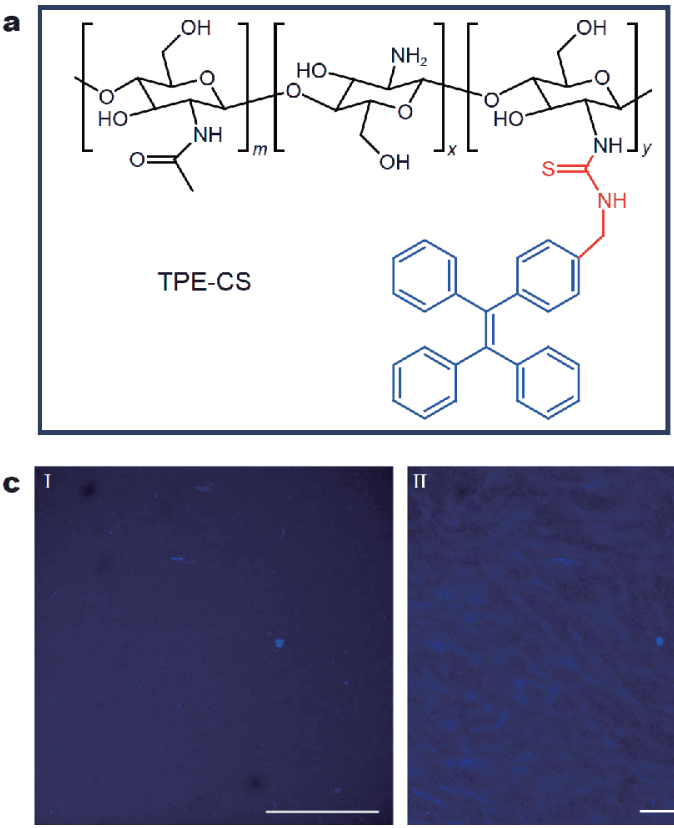

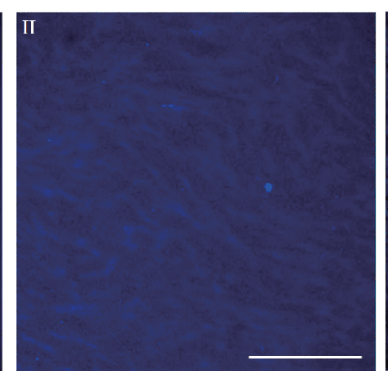

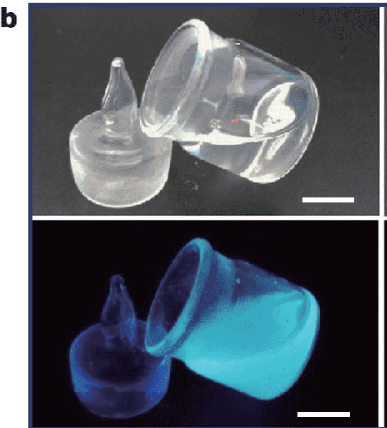
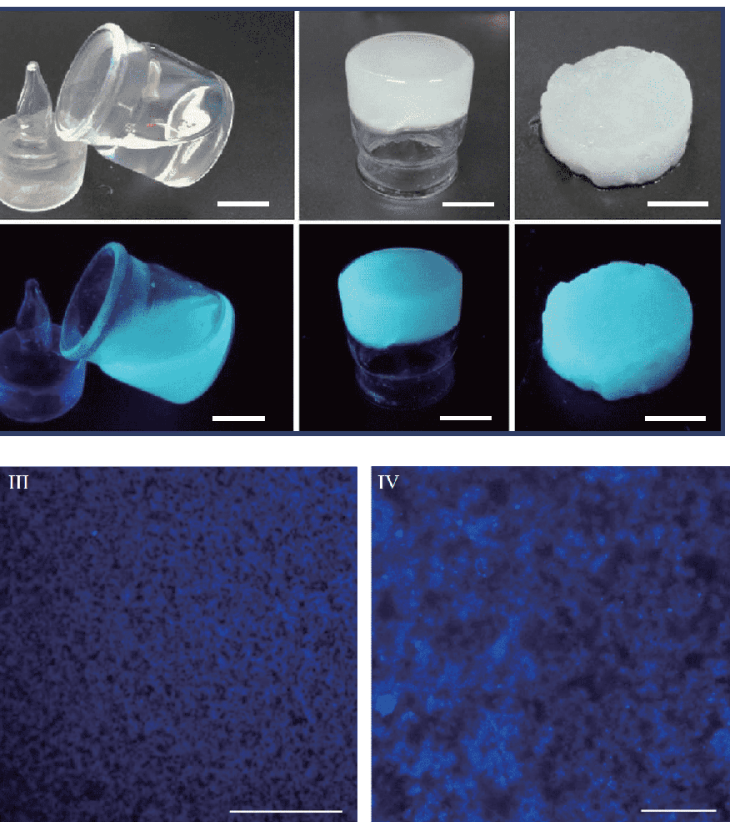

Figure 3 Real-time and in situ visualization of gelation process of CS by using AIEgen as fluorescent probe. (a) Molecular structure of TPE-CS. (b) Digital images of formation of hydrogel with TPE-CS: solution of TPE-CS, TPE-CS gel after thermal gelation, and TPE-CS hydrogel after complete removal of $\mathrm{LiOH}$ and urea; scale bar, $5.0 \mathrm{~mm}$. The fluorescent photographs were taken under ultraviolet illumination (365 nm). (c) Confocal laser scanning FL microscope images of gelation process of TPE-CS. (I) Solution; (II) gel after thermal gelation; (III, IV) hydrogel after rinsing. Scale bar, $250 \mathrm{~mm}$ for (I-III); $25 \mathrm{~mm}$ for (IV). Reproduced with permission from Ref. [18]. Copyright 2016, Nature Publishing Group.

fluorescent image of the solution shows no specific patterns (I). However, some bright areas start to appear as heating starts, corresponding to the thermal gelation stage. The bright areas continue developing over time. Then, the development slows down, and finally the pattern stabilizes (II), implying that the thermal gelation of the system possesses a certain terminal point. The gel structure evolves further after $\mathrm{LiOH}$ and urea were completely removed by rinsing (III), suggesting that a further gelation process occurs after the terminal point of thermal gelation. After the rinsing stage, the bright areas subdivide and contract, and dark textures form. Eventually, a reticular structure is generated in the final CS hydrogel (IV). Combined with an analysis of the mechanical, dynamic mechanical property measurements and X-ray diffraction patterns, a three-stage gelation model was proposed: (1) thermal-induced formation of inter/intra-macromolecular hydrogen bonds, (2) microcrystallization-induced junction generation and primary gel formation, and (3) further formation of hydrogen bonding and final CS hydrogel formation due to the removal of $\mathrm{LiOH}$ and urea. This study represents a unique attempt to visualize the entire gelation process of the CS $\mathrm{LiOH}$-urea aqueous system by using the AIE-active fluorescent probe. A detailed mechanism for the gelation process was demonstrated, which shed new light on the intermolecular interactions in complex biomacromolecular systems. With the growth of the AIE and biomaterials field, various AIE fluorogens could be used to visualize various gelation systems.

The motions of the polymer chains or segments with temperature changing involve a centurial and global problem-the glass transition problem [19], which can be considered as the case of a pure and glassy (or amorphous) polymer without any solvent. Glass transition temperature $\left(T_{\mathrm{g}}\right)$, is a fundamental physical and structural parameter that is related to various physical properties of a polymer. Thus, each physical property change during glass transition provides a measurement method for $T_{\mathrm{g}}$ determination. These methods, such as differential scanning calorimetry, dilatometry, and dynamic mechanical analysis, rely on the integral change because glass transition is an intrinsic phase transition. On the molecular level, $T_{\mathrm{g}}$ defines a threshold temperature at which the motion of the segments in polymer chains is liberated or frozen by a heating or cooling thermal treatment. In principle, such a thermal-induced process can be sensed by an AIE-active fluorescent probe because the activated segment motion pushes the motions of the probe molecules and then deactivates the FL emission or weakens the 
emission intensity if AIE-active molecules exist in the polymer matrix.

This assumption was validated by a rational experimental design, which was reported by Qiu et al. [20] in 2017. The researchers named their $T_{\mathrm{g}}$ detection technique as ADEtect, which combined the terms "AIE" and "detect". As shown in Fig. 4a, the setup and measurement procedures are simple. TPA-BMO and polystyrene (PS) were used as AIE-active fluorescent probe and polymer matrix, respectively. The PS film with $1 \mathrm{wt} \%$ TPA-BMO was prepared according to the standard procedure. The fluorescent images were captured by a charge-coupled device camera in a preset temperature window, and representative photos were selected as shown in Fig. 4b. The images showed that the emission intensity or brightness decreased with rising temperature.

To quantify the information captured by fluorescent images, the red, green, and blue values were obtained using Matlab, by which the brightness of an image was quantified as grayscale $(G)$ (Fig. $4 c$ ) in a standard formulation. The relative value $\left(G / G_{0}\right)$ against temperature was plotted (in Fig. 4 d, where $G_{0}$ was the grayscale at time $0 \mathrm{~s})$. The curve of $G / G_{0}$ declines steadily with temperature, and the point at which a slope change occurs is assigned to $T_{\mathrm{g}}$. Using the relative FL intensity $\left(I / I_{0}\right)$ seems to be a direct method, but in fact, the slope change on the $I / I_{0}$ curve is usually more ambiguous than that on the $G /$ $G_{0}$ curve. The reason for this accuracy improvement is that the full spectrum is recorded in the fluorescent image and calculated as $G / G_{0}$, but for $I / I_{0}$, only the peak intensity at a certain wavelength is analyzed, thereby leading to partial loss of spectral information. Based on the description, ADEtect has shown a series of advantages, including easy operation, high sensitivity, good reliability, and low cost. More importantly, this new method allows the measurement of multiple samples in parallel, showing great potential for high-throughput $T_{\mathrm{g}}$ measurement. Thus, ADEtect has demonstrated its great potential in the academic and industrial fields.

Besides the application in the study of chain movements of classical polymers, copolymers, and biomacromolecules, AIEgens have been also used in investigating dynamic polymers. These polymers, which are constructed through the self-assembly of supramolecular building blocks, have received considerable attention in recent years [21]. This type of polymers often exhibits hierarchically ordered structures, high stimuli responsiveness, and smart functions. Understanding the dynamic assembly process is important, but we are short of such techniques. Although scanning electron microscope, transmittance electron microscope, and atomic force microscope can help us view the microstructure and morphology at nanometer level, the information that corresponds to the end products or final assemblies, and the initial step and kinetic stimuli-responsive behavior of self-assemblies have hardly been visualized. A unique
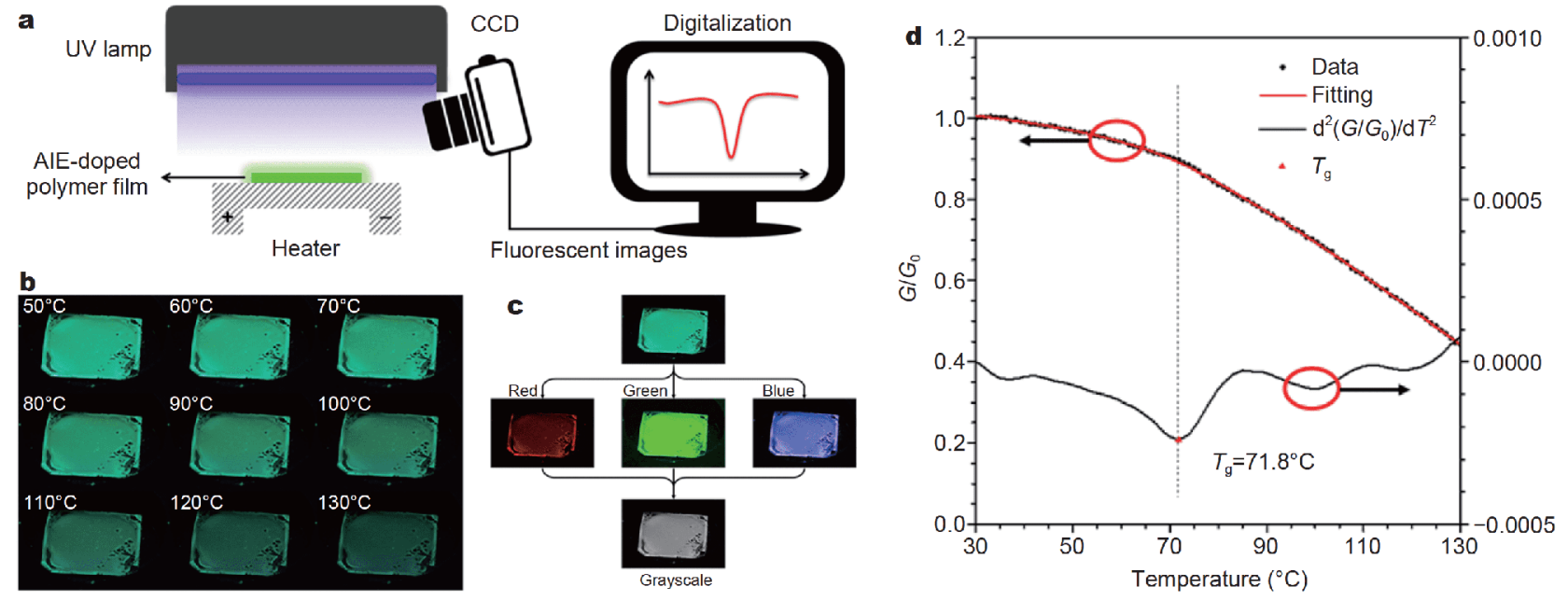

Figure 4 (a) Schematic of equipment setup of ADEtect measurement system. (b) Photographs of TPA-BMO-doped PS-1 films taken at different temperatures (from 50 to $130^{\circ} \mathrm{C}$ ). (c) Illustration of data processing method. (d) Change of relative grayscale $\left(G / G_{0}\right.$ ) of TPA-BMO-doped PS-1 film with temperature and associated fitting curve as well as second derivative of the fitting curve to reveal the change in $G / G_{0}$ at different temperatures. Heating rate: $6^{\circ} \mathrm{C} \mathrm{min}{ }^{-1} . G_{0}$ is the grayscale at time of $0 \mathrm{~s}$, and $G$ is calculated according to the equation of $G=0.2989 * \operatorname{Red}+0.5870 * \mathrm{Green}+$ $0.1140 *$ Blue. The inset shows the molecular structure of fluorescent probe TPA-BMO. Reproduced with permission from Ref. [20]. Copyright 2017, American Chemistry Society. 
characteristic of the AIE-active fluorescent probe is that it can dynamically change the emission intensity. Thus, it is an ideal probe to monitor the self-assembly process.

Recently, Tang et al. [22] reported visualizing the initial step of self-assembly process and phase transition by using stereogenic and amphiphilic AIE-active molecules (Z)-TPE-OEG and (E)-TPE-OEG (Fig. 5a), which were constructed by a hydrophobic TPE core and six thermoresponsive hydrophilic oligoethylene glycol (OEG) monomethyl ether chains. (Z)-TPE-OEG molecules selfassemble into vesicles, and $(E)$-isomers form micelles in water (Fig. 5a). The initial step of their self-assembly processes can be visualized based on AIE characteristics, with a sensitivity much higher than that of the method based on transmittance measurement. A continuous phase transition from microscopic self-assembly to macroscopic aggregation has been recorded by the $(Z)$-type amphiphile due to the intrinsic thermo-responsive behavior of OEG chains. The entire process can be successfully visualized in situ by confocal laser scanning microscopy (CLSM). Based on the analysis of the fluorescent images, the kinetic process of the self-assembly shows different stages (Fig. 5b), which can never be observed by examining the appearance of precipitates (FL and CLSM images in Fig. 5a).

These instructive studies show the uniqueness of AIEactive fluorescent probes. Distinct from the traditional fluorescent probes, the fluorescent probes characterized by AIE activity are not emissive lookers that simply stand by and report the events, but rather actively participate in the events. These probes can sense the emancipated segment motions in solid amorphous polymer matrix by concomitantly activated intramolecular rotations and vibrations and report the glass transition temperature.

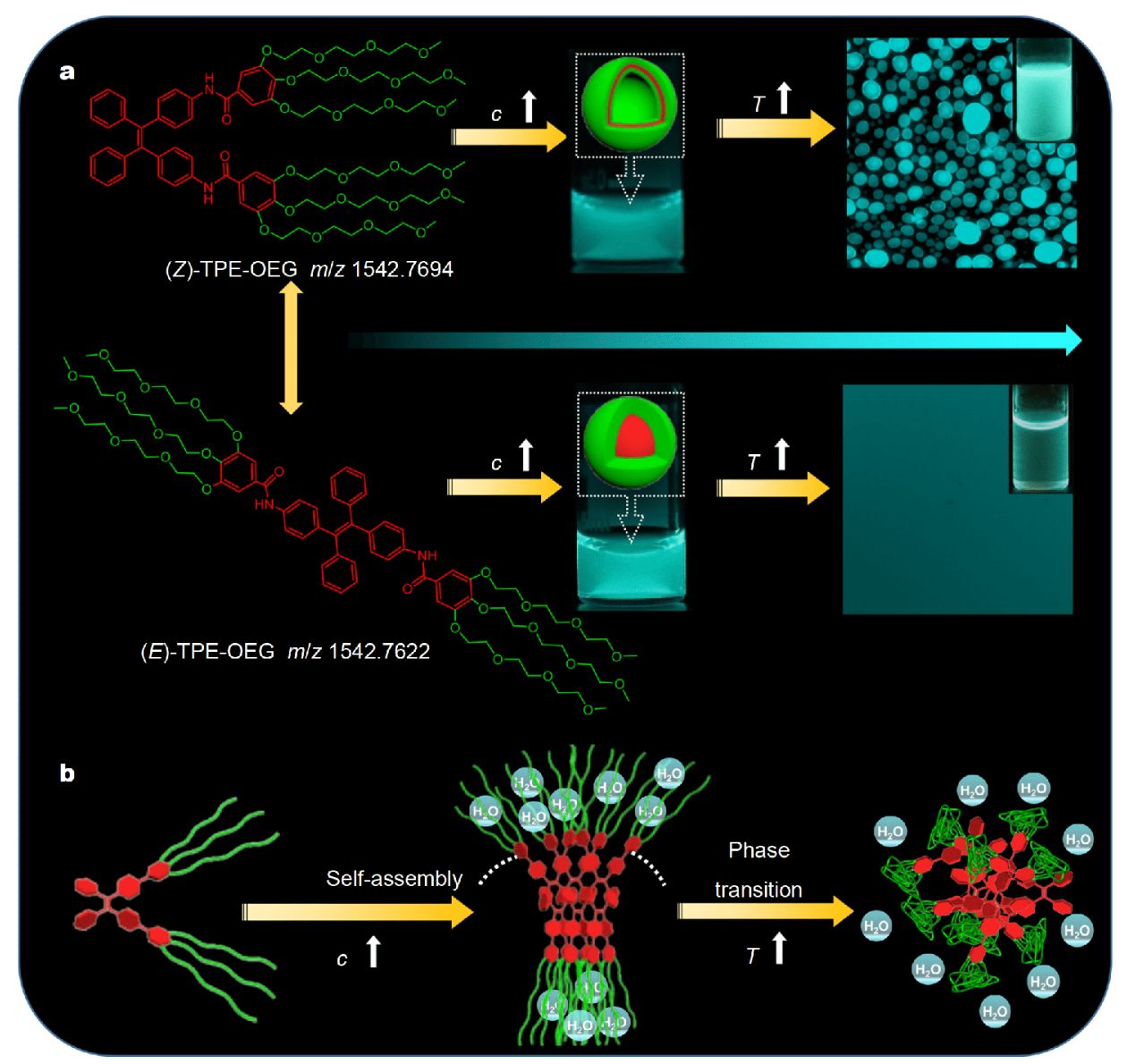

Figure 5 (a) Molecular structures of (Z)-TPE-OEG and (E)-TPE-OEG and visualization of their self-assembly and thermo-responsive behaviors. (b) Illustration of self-assembly and phase transition of (Z)-TPE-OEG. As the temperature increases, packing morphology of $(Z)$-amphiphiles changes from vesicles to large aggregates due to dehydration of OEG chains. $c$ : concentration, and $T$ : temperature. The photographs in (a) show the microstructures formed in the aqueous solution taken by CLSM and digital camera. Reproduced with permission from Ref. [22]. Copyright 2019, American Chemistry Society. 
These probes can localize in the nano-scale networks of polymeric microgels and in situ sense the cononsolvency effect by the enhancement of their emission. Furthermore, these probes can be labeled to the polysaccharide side chains to monitor the CS hydrogel formation process in an in situ and real-time manner, and to fluorescently illustrate a step-by-step scheme of H-bond formation, microcrystalline generation, urea- $\mathrm{LiOH}$ release, and further shrinkage.

With such an unmatchable nature, AIE-active fluorescent probes may shed light on a greater number of specific spaces that are dark and deep. For example, AIEgens are assumed to be the best candidates to visualize interface structures and monitor the interfacial dynamic processes because the restriction and liberation of intramolecular motion usually occur at the interface. Some recent publications have shown the potential of this research area [23-26]. According to the restriction of intramolecular motion mechanism of AIE activities, various external stimuli can easily interfere with the sensing of dynamic processes. Subtle changes in microenvironment can be reflected in FL fluctuation. Incorporation with other signals besides emission intensity is a valuable method that has been validated in studies in which the dichroism signal has been engaged to monitor the dynamic process in an in situ and real-time manner [27-30]. Considering the importance of visually monitoring biological events in life science and that the relevant processes occur under tissues or inside organisms, we find that the AIEgens brightly emitting in near-infrared II region represent a highly demanding subject that will become a thriving research area.

Received 15 March 2019; accepted 21 May 2019; published online 19 June 2019

1 Hong Y, Lam JWY, Tang BZ. Aggregation-induced emission. Chem Soc Rev, 2011, 40: 5361

2 Guo J, Zhao Z, Tang BZ. Purely organic materials with aggregation-induced delayed fluorescence for efficient nondoped OLEDs. Adv Opt Mater, 2018, 6: 1800264

3 Gao M, Tang BZ. Fluorescent sensors based on aggregation-induced emission: Recent advances and perspectives. ACS Sens, 2017, 2: 1382-1399

4 Chen S, Wang H, Hong Y, et al. Fabrication of fluorescent nanoparticles based on AIE luminogens (AIE dots) and their applications in bioimaging. Mater Horiz, 2016, 3: 283-293

5 Mei J, Hong Y, Lam JWY, et al. Aggregation-induced emission: The whole is more brilliant than the parts. Adv Mater, 2014, 26: 5429-5479

6 Lim XZ. The nanolight revolution is coming. Nature, 2016, 531: 26-28

7 Qi J, Chen C, Ding D, et al. Aggregation-induced emission lumi- nogens: Union is strength, gathering illuminates healthcare. Adv Healthcare Mater, 2018, 7: 1800477

8 Mei J, Leung NLC, Kwok RTK, et al. Aggregation-induced emission: Together we shine, united we soar! Chem Rev, 2015, 115: 11718-11940

9 Zhang H, Zheng X, Kwok RTK, et al. In situ monitoring of molecular aggregation using circular dichroism. Nat Commun, 2018, 9: 4961

10 Mukherji D, Marques CM, Kremer K. Polymer collapse in miscible good solvents is a generic phenomenon driven by preferential adsorption. Nat Commun, 2014, 5: 4882-4887

11 Zhang G, Wu C. Reentrant coil-to-globule-to-coil transition of a single linear homopolymer chain in a water/methanol mixture. Phys Rev Lett, 2001, 86: 822-825

12 Tanaka F, Koga T, Winnik FM. Temperature-responsive polymers in mixed solvents: Competitive hydrogen bonds cause cononsolvency. Phys Rev Lett, 2008, 101: 028302

13 Xue J, Bai W, Duan $\mathrm{H}$, et al. Tetraphenylethene cross-linked thermosensitive microgels via acylhydrazone bonds: Aggregationinduced emission in nanoconfined environments and the cononsolvency effect. Macromolecules, 2018, 51: 5762-5772

14 Ladet S, David L, Domard A. Multi-membrane hydrogels. Nature, 2008, 452: 76-79

15 Aider M. Chitosan application for active bio-based films production and potential in the food industry: Review. LWT-Food Sci Tech, 2010, 43: 837-842

16 Kozicki M, Kołodziejczyk M, Szynkowska M, et al. Hydrogels made from chitosan and silver nitrate. Carbohyd Polym, 2016, 140: $74-87$

17 Li C, Han Q, Guan Y, et al. Thermal gelation of chitosan in an aqueous alkali-urea solution. Soft Matter, 2014, 10: 8245-8253

18 Wang Z, Nie J, Qin W, et al. Gelation process visualized by aggregation-induced emission fluorogens. Nat Commun, 2016, 7: 12033

19 Couzin J. How much can human life span be extended? Science, 2005, 309: 83

20 Qiu Z, Chu EKK, Jiang M, et al. A simple and sensitive method for an important physical parameter: Reliable measurement of glass transition temperature by AIEgens. Macromolecules, 2017, 50: 7620-7627

21 Jiang L, Cao S, Cheung PPH, et al. Real-time monitoring of hydrophobic aggregation reveals a critical role of cooperativity in hydrophobic effect. Nat Commun, 2017, 8: 15639

22 Peng HQ, Liu B, Wei P, et al. Visualizing the initial step of selfassembly and the phase transition by stereogenic amphiphiles with aggregation-induced emission. ACS Nano, 2019, 13: 839-846

23 He Z, Liu P, Zhang S, et al. A freezing-induced turn-on imaging modality for real-time monitoring of cancer cells in cryosurgery. Angew Chem Int Ed, 2019, 58: 3834-3837

24 Alam P, Leung NLC, Cheng Y, et al. Spontaneous and fast molecular motion at room temperature in the solid state. Angew Chem Int Ed, 2019, 58: 4536-4540

25 Guan W, Wang S, Lu C, et al. Fluorescence microscopy as an alternative to electron microscopy for microscale dispersion evaluation of organic-inorganic composites. Nat Commun, 2016, 7: 11811

26 Qiu Z, Zhao W, Cao M, et al. Dynamic visualization of stress/strain distribution and fatigue crack propagation by an organic mechanoresponsive AIE luminogen. Adv Mater, 2018, 30: 1803924

27 Song F, Xu Z, Zhang Q, et al. Highly efficient circularly polarized 
electroluminescence from aggregation-induced emission luminogens with amplified chirality and delayed fluorescence. Adv Funct Mater, 2018, 28: 1800051

28 Xiong JB, Feng HT, Sun JP, et al. The fixed propeller-like conformation of tetraphenylethylene that reveals aggregation-induced emission effect, chiral recognition, and enhanced chiroptical property. J Am Chem Soc, 2016, 138: 11469-11472

29 Zhang J, Liu Q, Wu W, et al. Real-time monitoring of hierarchical self-assembly and induction of circularly polarized luminescence from achiral luminogens. ACS Nano, 2019, 13: 3618-3628

$30 \mathrm{Qu} \mathrm{H}$, Wang Y, Li Z, et al. Molecular face-rotating cube with emergent chiral and fluorescence properties. J Am Chem Soc, 2017, 139: 18142-18145

Acknowledgements This work was financially supported by the National Natural Science Foundation of China (51573158 and 21094571), the National Program for Support of Top-Notch Young Professionals, the Innovation and Technology Commission of Hong Kong (ITCCNERC14SC01); the Research Grants Council of Hong Kong (16308016, C2014-15G, C6009-17G and A-HKUST605/16), and the Technology Plan of Shenzhen (JCYJ20160229205601482).

Author contributions Jiang $\mathrm{N}$ and Shen $\mathrm{T}$ contributed equally to the reference collection and manuscript writing; Sun JZ contributed to the text correction, figure modification and general discussion; Tang BZ contributed the materials selection and scientific significance recognition.

Conflict of interest The authors declare no conflict of interest.

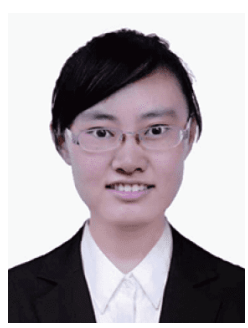

Nan Jiang received her BSc degree from Hebei University in 2010. She conducted her graduate study and obtained her Master's degree in polymer chemistry and physics in 2014. She is currently a $\mathrm{PhD}$ candidate at Zhejiang University under the supervision of Prof. Ben Zhong Tang and Prof. Jing Zhi Sun. Her research focuses on the development of new functional AIE materials.

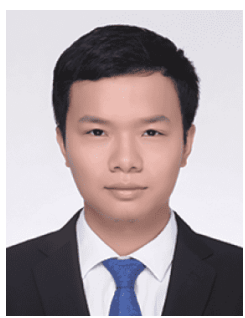

Tanxiao Shen is a $\mathrm{PhD}$ candidate at the Department of Polymer Science and Engineering at Zhejiang University. After receiving BEng and MEng degrees from Sichuan University in 2014 and 2017, respectively, he joined Prof. Jing Zhi Sun and Prof. Ben Zhong Tang's group. His research interest includes the synthesis and applications of functional polyacetylenes and materials with AIE characteristics.

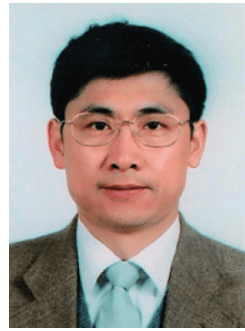

Jing Zhi Sun is a professor at the Department of Polymer Science and Engineering at Zhejiang University. He received $\mathrm{BSc}, \mathrm{MSc}$, and $\mathrm{PhD}$ degrees from Jilin University. In 1986 and 1996, he worked at Liaoyang Petroleum \& Fiber Co. Ltd. and obtained his engineering license in 1991. After postdoctoral research at the College of Materials and Chemical Engineering at Zhejiang University, he joined the university as an assistant professor in 2001 and was promoted to professor in 2006. His research interest includes AIE mechanism, materials, and applications; design and synthesis of functional polyacetylenes; assembly of organic $\pi$-conjugated moieties and inorganic semiconductor nanostructures; and fundamental research on charge generation, photoconductivity, and charged carrier transport in organic solids.

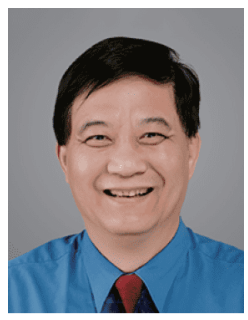

Ben Zhong Tang is Stephen K. C. Cheong Professor of Science, Chair Professor of Chemistry, and Chair Professor of Chemical and Biological Engineering at The Hong Kong University of Science and Technology (HKUST). He received $\mathrm{BSc}$ and $\mathrm{PhD}$ degrees from South China University of Technology and Kyoto University, respectively. He conducted postdoctoral research at the University of Toronto, joined HKUST as an assistant professor in 1994, and was promoted to chair professor in 2008. He was elected to the Chinese Academy of Sciences and the Royal Society of Chemistry in 2009 and 2013, respectively. His research interest includes macromolecular chemistry, materials science, and biomedical theranostics. He is spearheading the research on AIE, a topic ranked second in Chemistry and Materials Science by Thomson Reuters in its report on Research Fronts 2015 .

\section{在哪里运动受限, 就在哪里发光}

姜南 $^{1 \dagger}$, 沈谈笑 ${ }^{1 \dagger}$, 孙景志 ${ }^{1^{*}}$, 唐本忠 ${ }^{1,2,3^{*}}$

摘要 本文通过典型研究实例列举了具有聚集诱导发光(AIE)性能 的苂光探针分子的一些新用途, 如监测分子聚集体形成细节、双 亲高分子链的共非溶剂效应、壳聚糖溶液中的多步溶胶-凝胶转 变、测量玻璃化转变温度的新方法以及原位显示动态高分子组装 过程的初始阶段等. 在这些研究中, AIE-型荧光探针充分显示了其 原位、实时、动态变化大和对比度高的特点, 揭示了其他研究手 段难以提供的动态信息细节. 这类工作机理独特的荧光探针将在 未来的材料结构与性能的内在关系研究中发挥越来越重要的作用. 